\title{
Innovative drug nanocarriers by incorporating thermoresponsive polymer in phospholipid bilayers
}

\author{
Anna Tzani ${ }^{1}$, Nikolaos Naziris ${ }^{1}$, Natassa Pippa ${ }^{1,2}$, Stergios Pispas ${ }^{2}$ and Costas Demetzos ${ }^{1 *}$ \\ ${ }^{1}$ Section of Pharmaceutical Technology, Department of Pharmacy, School of Health Sciences, National and Kapodistrian University of Athens, Panepistimioupolis \\ Zografou, Athens, Greece \\ ${ }^{2}$ Theoretical and Physical Chemistry Institute, National Hellenic Research Foundation, 48 Vassileos Constantinou Avenue, Athens, Greece
}

\begin{abstract}
A liposome with stimuli-responsive properties, would enable us to address several systemic and intracellular delivery barriers. The aim of this study was to combine the thermoresponsive polymer $\mathrm{C}_{12} \mathrm{H}_{25}$-poly(N-isopropylacrylamide)-COOH $\left(\mathrm{C}_{12} \mathrm{H}_{25}-\mathrm{PNIPAM}-\mathrm{COOH}\right)$ with the phospholipid L- $\alpha$-phosphatidylcholine hydrogenated (Soy) (HSPC) into chimeric/mixed vesicles and to investigate the resultant structures by light scattering techniques and differential scanning calorimetry (DSC). Liposome thermo-responsiveness occurs due to the PNIPAM segment exhibiting a lower critical solution temperature (LCST) at approximately $32^{\circ} \mathrm{C}$, in aqueous solutions, which alters its hydrophilic-to-hydrophobic balance. After the physicochemical study, it was deduced that the biomaterials' molar ratio was determinant for colloidal stabilization of the lyotropic nanosystems. Also, thermodynamic findings are in line with the physicochemical results, since the presence of polymer resulted in different thermodynamic content, which corresponds to a different thermotropic behavior, compared with pure lipid bilayers. With greater understanding of these differences, a wide range of innovative liposomal nanocarriers could be developed in the future, for drug and gene delivery
\end{abstract}

\section{Introduction}

Liposomes are nanovesicles, composed of at least one lipid bilayer. Their building blocks are amphiphilic lipids, usually phospholipids, which have been characterized as liquid crystals, an intermediate phase that exhibits properties between those of solid crystals and isotropic liquids. Phospholipids become hydrated in aqueous forms and create lamellar phases. The $\mathrm{L}_{\alpha}$ mesophase refers to liquid crystal phase, while $L_{\beta}$ is a gel or solid crystal phase. The transition $L_{\beta} \rightarrow L_{\alpha}$ occurs when temperature is ascending, so the aligning forces become weaker and lipids accelerate [1-3].

Thermoresponsive polymers are macromolecules that exhibit an excessive and reversible change in their physicochemical properties, when temperature is altered [4]. The key characteristic of thermoresponsive polymersis their ability to change their hydrophilicity around certain temperature values. They exhibit a two-phase region, in which the polymer is found in both hydrophilic and hydrophobic form. Depending on whether that region is found at high or low temperatures, an upper or lower critical solution temperature (UCST or LCST) exists, respectively. Research mainly focuses on polymers that exhibit LCST in aqueous solution, for medical reasons $[5,6]$.

Development of chimeric/mixed liposomes, which arise from the combination of different in nature biomaterials, such as phospholipids and polymers, gives the opportunity to generate advanced drug delivery nanosystems (aDDnSs) [7]. These complex self-assembled nanostructures act as facilitators for the creation of innovative therapeutic nanosystems and respond to the requirements of recent trends in nanomedicine, as indicated by systems pharmacology [8-13].

The aim of this study is to design and develop thermoresponsive chimeric liposomes, based on a phospholipid and a thermoresponsive homopolymer and to investigate their thermotropic and physicochemical characteristics. Such systems can use temperature fluctuations as a trigger to rearrange and release the drug molecule, an ability that makes them more efficient in site-specific drug delivery than conventional liposomes.

\section{Materials and methods}

\section{Materials}

The phospholipid used for liposomal formulations was L- $\alpha$ phosphatidylcholine, hydrogenated (Soy) (HSPC) (Figure 1a). It was purchased from Avanti Polar Lipids Inc., (Albaster, AL, USA) and used without further purification. Chloroform and all other reagents used were of analytical grade and purchased from Sigma-Aldrich Chemical Co. $\mathrm{C}_{12} \mathrm{H}_{25}$-PNIPAM-COOH was synthesized by RAFT polymerization using 2-(dodecylthiocarbonothioylthio)-2-methylpropionic acid as the RAFT agent, and AIBN as the radical initiator in 1,4-dioxane. The molecular weight of the synthesized homopolymer was 20,000 and had narrow molecular weight distribution (Figure 1b). The structures of the components of bio-inspired nanostructures are presented in Figure 1.

\section{Preparation of liposomes}

Conventional and chimeric liposomal drug nanocarriers were prepared by the thin-film hydration method. Briefly, appropriate amounts of HSPC and HSPC: $\mathrm{C}_{12} \mathrm{H}_{25}$-PNIPAM-COOH (9:0.0, 9:0.02, 9:0.05, 9:0.1, 9:0.2 and 9:0.5 molar ratios) mixtures were dissolved in

Correspondence to: Costas Demetzos, Section of Pharmaceutical Technology, Department of Pharmacy, School of Health Sciences, National and Kapodistrian University of Athens, Panepistimioupolis Zografou, 15771 Athens, Greece, Tel: +30 2107274596; Fax: +30 2107274027; E-mail: demetzos@pharm.uoa.gr

Key words: chimericliposome, lipid, polymer, thermoresponsiveliposome

Received: April 11, 2017; Accepted: May 08, 2017; Published: May 11, 2017 
a.<smiles>CCCCCCCCCCCCCCCCCCCCCCCCCCC(=O)OCC(O)COP(=O)([O-])OCC[N+](C)(C)C</smiles>

b.

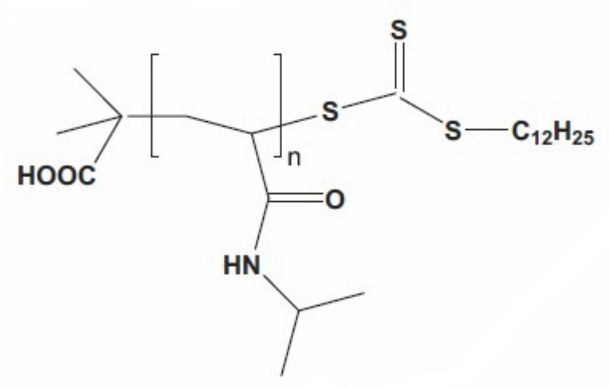

Figure 1. Chemical structures of(a) HSPC lipid and (b) $\mathrm{C}_{12} \mathrm{H}_{25}$-poly(N-isopropylacrylamide)$\mathrm{COOH}$.

chloroform and then transferred into a round flask connected to a rotary evaporator. Vacuum was applied and the mixed phospholipid/ polymer thin film was formed by slow removal of the solvent at $50^{\circ} \mathrm{C}$. The film was maintained under vacuum, for at least $24 \mathrm{~h}$, in a desiccator, to remove traces of solvent and subsequently, it was hydrated in phosphate buffer saline (PBS) ( $\mathrm{pH}=7.4)$. Lipid films were hydrated in HPLC-grade water, by slowly stirring for $1 \mathrm{~h}$, in a water bath, above the phase transition of lipids $\left(53^{\circ} \mathrm{C}\right)$. The resultant multilamellar vesicles (MLVs) were subjected to three 10 min sonication cycles (amplitude 70, cycle 0.5 ), interrupted by a 10 min resting period, in water bath, using a probe sonicator (UP 200S, Dr. Hielsher GmbH, Berlin, Germany). The resultant vesicles were allowed to anneal for $30 \mathrm{~min}$.

\section{Mean size, size distribution and zeta potential}

The effect of PNIPAM on the liposomal lipid bilayers was evaluated by measuring the size (hydrodynamic diameter, $\mathrm{D}_{\mathrm{h}}$ ), polydispersity index (PDI) and zeta potential ( $\zeta$-pot) of liposomes. The $\mathrm{D}_{\mathrm{h}}$, PDI and $\zeta$-pot of the particles were investigated by dynamic and electrophoretic light scattering (DLS and ELS) and used for the characterization of the liposomal dispersions immediately after preparation ( $\mathrm{t}=0$ days), as well as for the monitoring of their physical stability over time ( $t=31$ days, at $4^{\circ} \mathrm{C}$, in liquid form). For each measurement, $100 \mu \mathrm{L}$ aliquots were 30 fold diluted in HPLC-grade water. Measurements were performed at a detection angle of $90^{\circ}$ and at $25^{\circ} \mathrm{C}$, in a photon correlation spectrometer (Zetasizer 3000 HSA, Malvern, UK) and analyzed by CONTIN method (MALVERN software).

\section{Preparation of pure and chimeric lipid bilayers}

Pure lipid and chimeric bilayers were prepared by mixing the appropriate amounts of HSPC and $\mathrm{C}_{12} \mathrm{H}_{25}$-PNIPAM in chloroform/ methanol $(9: 1 \mathrm{v} / \mathrm{v})$ solutions and the subsequent evaporation of the solvents under vacuum and heat. Briefly, stock solutions were prepared by dissolving the homopolymer in chloroform/methanol $(9: 1 \mathrm{v} / \mathrm{v})$. Appropriate amounts of these solutions were mixed with $30.0 \mathrm{mg}$ of HSPC, in order to obtain the desirable molar ratios (9:0.02, 9:0.05, 9:0.1, 9:0.2 and 9:0.5) and the solutions were transferred into vials. Then, the vials were placed into a vacuum machine (TechneDri-Block DB-3 Thermostat Teche Sample Concentrator). Chimeric phospholipid/ homopolymer films were formed by removing the solvent at $50^{\circ} \mathrm{C}$. The films were maintained under vacuum for $2 \mathrm{~h}$ and then, in a desiccator, for at least $24 \mathrm{~h}$, in order to remove traces of solvent. The obtained laminated bilayers were hydrated into the appropriate aqueous medium and then studied by DSC.

\section{Differential scanning calorimetry (DSC)}

DSC experiments were performed on an 822eMettler-Toledo (Schwerzenbach, Switzerland) calorimeter calibrated with pure indium $\left(\mathrm{T}_{\mathrm{m}}=156.6^{\circ} \mathrm{C}\right)$. Sealed aluminum $40 \mu \mathrm{l}$ crucibles were used as sample holders. The samples investigated were HSPC and HSPC: $\mathrm{C}_{12} \mathrm{H}_{25}$ PNIPAM (9:0.02; 9:0.05, 9:0.1, 9:0.2 and 9:0.5 molar ratios) lipid bilayers with a $10 \mathrm{mg} / \mathrm{ml}$ concentration (with reference to the whole dispersion) for the overall lipid content. An empty aluminum crucible was used as reference. Prior to measurements the crucibles were subjected to a temperature over the transition of $\operatorname{HSPC}\left(53.6^{\circ} \mathrm{C}\right)$ to ensure equilibration. All samples were scanned repeatedly until identical thermograms were obtained. Two cooling-heating cycles were performed; 10 to $60^{\circ} \mathrm{C}$ at $2^{\circ} \mathrm{C} / \mathrm{min}$ scanning rate. The second heating run was taken into account. Enthalpy changes and characteristic transition temperatures were calculated with Mettler-Toledo STAR software.

\section{Statistical analysis}

Results are shown as mean value \pm standard deviation (S.D.) of three independent measurements. Statistical analysis was performed using Student's t-test and multiple comparisons were done using oneway ANOVA. P-values $<0.05$ were considered statistically significant. All statistical analyses were performed using "EXCELL".

\section{Results and discussion}

\section{Physicochemical characterization of HSPC: $\mathrm{C}_{12} \mathrm{H}_{25}$-PNIPAM- $\mathrm{COOH}$ liposomes}

Physicochemical characteristics of conventional HSPC liposomes and of thermoresponsive polymer-grafted HSPC: $\mathrm{C}_{12} \mathrm{H}_{25}$-PNIPAM$\mathrm{COOH}$ liposomes in different molar ratios (9:0.0, 9:0.02, 9:0.05, 9:0.1, 9:0.2 and 9:0.5 molar ratios) are presented in Table 1. It should be noted that PBS was chosen as the dispersion medium because the $\mathrm{pH}(\sim 7.4)$ and the ionic strength of PBS resemble the conditions met within the human body.

The size of HSPC liposomes in PBS was around $100 \mathrm{~nm}$, which is in agreement with previous publications. Their $\zeta$-potential was close to zero, because of the absence of net charge on the nanocarrier surface [14].

Conventional HSPC liposomes (reference system) exhibit the smaller size and polydispersity than the other prepared systems. The $\mathrm{C}_{12} \mathrm{H}_{25}$-PNIPAM-COOH has a small hydrophobic alkyl chain, which is grafted into the liposomal. After the incorporation of the polymer at the lowest ratio, a great increase of $D_{h}$ was observed compared

Table 1. The physicochemical characteristics of chimeric HSPC: $\mathrm{C}_{12} \mathrm{H}_{25}-\mathrm{PNIPAM}-\mathrm{COOH}$ liposomes.

\begin{tabular}{|l|c|c|c|c|c|c|c|}
\hline System & Molarratio & $\mathbf{D}_{\mathbf{h}}{ }^{\mathbf{1}} \mathbf{( n m )}$ & $\mathbf{S D}^{\mathbf{2}}$ & PDI $^{\mathbf{3}}$ & $\mathbf{S D}$ & $\begin{array}{c}\zeta-\mathbf{p o t}^{\mathbf{4}} \\
\mathbf{( m V )}\end{array}$ & $\mathbf{S D}$ \\
\hline HSPC & - & 100.7 & 1.4 & 0.466 & 0.006 & 2.2 & 0.4 \\
\hline HSPC $: \mathrm{C}_{12} \mathrm{H}_{25}$-PNIPAM-COOH & $9: 0.02$ & 378.9 & 25.1 & 1.000 & 0.000 & -6.0 & 1.4 \\
\hline HSPC $: \mathrm{C}_{12} \mathrm{H}_{25}$-PNIPAM-COOH & $9: 0.05$ & 418.7 & 25.2 & 1.000 & 0.000 & -6.5 & 1.0 \\
\hline HSPC $: \mathrm{C}_{12} \mathrm{H}_{25}$-PNIPAM-COOH & $9: 0.1$ & 198.6 & 7.1 & 0.664 & 0.063 & -8.7 & 0.2 \\
\hline HSPC $: \mathrm{C}_{12} \mathrm{H}_{25}$-PNIPAM-COOH & $9: 0.2$ & 221.4 & 3.0 & 0.608 & 0.033 & -9.8 & 0.8 \\
\hline HSPC $: \mathrm{C}_{12} \mathrm{H}_{25}$-PNIPAM-COOH & $9: 0.5$ & 242.1 & 37.5 & 0.781 & 0.191 & -5.8 & 2.1 \\
\hline
\end{tabular}

${ }^{1}$ Hydrodynamic Diameter; ${ }^{2}$ Standard Deviation, ${ }^{3}$ Polydispersity Index; ${ }^{4} \zeta$-potential 
with pure HSPC liposomes. For molar ratios 9:0.02 and 9:0.05, $\mathrm{D}_{\mathrm{h}}$ was quadrupled and PDI had the maximum value (PDI=1), indicating the heterogeneity of nanoassemblies. By increasing the amount of $\mathrm{C}_{12} \mathrm{H}_{25}$-PNIPAM-COOH, better cooperativity between biomaterials was noticed. Specifically, in chimeric system 9:0.1, $D_{\mathrm{h}}$ was $198.6 \mathrm{~nm}$, which is the smallest size in chimeric liposomes, while higher molar ratios led to larger sizes, proportionally to PNIPAM's concentration. The last three colloidal systems (9:0.1, 9:0.2, 9:0,5 molar ratios) were of low size polydispersity, so their PDI followed the same trend with their $\mathrm{D}_{\mathrm{h}}$ (initial increase, but following acute decrease), as the percentage of $\mathrm{C}_{12} \mathrm{H}_{25}$-PNIPAM-COOH was increased.

Liposomal size is strongly dependent on polymer concentration, since HSPC phospholipids are lyotropic liquid crystals and modify their formation when temperature and/or concentration change. Even a small amount of $\mathrm{C}_{12} \mathrm{H}_{25}$-PNIPAM-COOH can cause membrane curvature, as long as it improves lateral pressure between lipids and due to repulsive forces among them and polymer chains. However, the higher the quantity of polymer, the easier for a liposomal membrane to curve [15].

Zeta potential is another important parameter that gives an indication, concerning the surface charge of liposomal nanovectors. The $\zeta$-potential of HSPC liposomes was found positive, but near zero, because of the absence of net charge on the liposomal membrane. After the incorporation of $\mathrm{C}_{12} \mathrm{H}_{25}$-PNIPAM-COOH, a reversal of $\zeta$-potential from positive to negative values was observed. As the molar ratio of the homopolymer was increased, the absolute value of $\zeta$-potential was increased too. This phenomenon was expected because, after the hydration of the HSPC: $\mathrm{C}_{12} \mathrm{H}_{25}$-PNIPAM-COOH nanosystems, the terminal $-\mathrm{COOH}$ group was ionized, since $\mathrm{PBS}$ has $\mathrm{pH}=7.4$ and the $\mathrm{pKa}$ of $-\mathrm{COOH}$ is around 4.5. These observations indicate that the polymeric chains induce some structural perturbations when included in liposomal membrane bilayers. However, the negative charge of $-\mathrm{COOH}$ group (-COO-) was so small, that the $\zeta$-potential remained below $-30 \mathrm{mV}$. This means that $\zeta$-potential cannot be considered as a remarkable factor of stability for these chimeric liposomal colloidal dispersions, but in any case, the experimental observations of this parameter indicate formation of chimeric lipid/polymer liposomes with the polymers chains in the periphery.

\section{Stability assessment of thermoresponsive polymer-grafted liposomes}

The physical stability over time of all conventional and chimeric nanosystems was assessed by measuring the size and size distribution

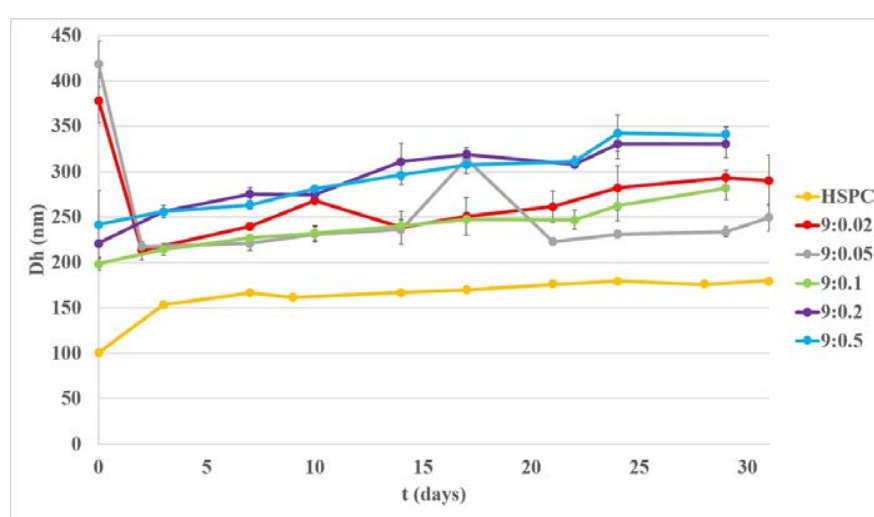

Figure 2. Stability studies in conventional and chimeric nanoassemblies, concerning their size $\left(\mathrm{Z}_{\text {Ave }}\right)$. overall one-month period. As shown in Figures 2 and 3, all liposomal formulations in PBS were found to roughly retain their original size, but most of them reached high PDI values at the end of the assessment (data not shown).

The smaller size was exhibited in conventional liposomes, which is maintained through weeks. Chimeric structures were separated in two groups. Lower molar ratios 9:0.02 and 9:0.05 belong to the first group and display the highest $\mathrm{D}_{\mathrm{h}}$, approximately $400 \mathrm{~nm}$, immediately after preparation, while the third day their size already reduced by half and then remained around so. The second group consists of the 9:0.1, 9:0.2 and 9:0.5 chimeric liposomes, exhibiting the minimum size during their preparation day ( $\mathrm{t}=0$ days), which is increased slightly, as days passed. As a consequence, it is concluded that 9:0.02 and 9:0.05 chimeric systems need longer relaxation time, in order to achieve better cooperativity among their biomaterials, while higher molar ratios develop adequate cooperativity during annealing time (30 min), right after sonication [16]. The lipid chains of HSPC are saturated and the $\mathrm{C}_{12} \mathrm{H}_{25}$ - is a small hydrophobic segment. These two conditions may explain the classification of chimeric liposomes in two categories, since the increase in the number of the incorporated chains within the liposome bilayers may result in spatial constrains and gradual morphological changes.

The HSPC: $\mathrm{C}_{12} \mathrm{H}_{25}$-PNIPAM-COOH chimeric liposomes were found to retain their original physicochemical characteristics, at least for the time period that they were studied. It is obvious that $\mathrm{C}_{12} \mathrm{H}_{25}$-PNIPAM-COOH provides colloidal stability to the systems, through various biophysical ways. Its hydrophilic tails $(-\mathrm{COOH})$ develop repulsive interactions, due to various effects, which keep the nanoassemblies in long distance. Steric stabilization includes the enthalpic process, during which the water molecules between opposing chains are driven away, leading to enthalpy increase and subsequent repulsion [17]. Furthermore, hydrated polymer chains prevent liposome aggregation, due to their stereochemical conformation. Both repulsive forces are responsible for keeping the chimeric nanovectors far enough, thus avoiding the van der Waals attraction of the DLVO theory, since there is an absence of significant electrostatic repulsion, which usually applies on colloidal dispersion systems [18].

\section{Thermotropic behavior investigation of $\mathrm{HSPC}: \mathrm{C}_{12} \mathrm{H}_{25}$ PNIPAM-COOH chimeric bilayers}

The DSC heating and cooling profiles of the prepared pure HSPC bilayers, as well as of the $\mathrm{C}_{12} \mathrm{H}_{25}$-PNIPAM-COOH-grafted HSPC bilayers in PBS are presented in Figure 4 and 5 respectively. The values of the corresponding thermodynamic parameters for the system phase transitions are shown in Tables 2 and 3. The effect of the presence and amount of the homopolymer upon the lipid membrane's thermotropic/ phase behavior is discussed, in terms of self-assembled organization, fluidity and cooperativity.

The neat HSPC bilayers exhibited two phase transitions, as the temperature was rising. The first was a wide pretransition, with negligible enthalpy change, from gel $\left(\mathrm{L}_{\beta}\right)$ to ripple phase $\left(\mathrm{P}_{\beta}\right)$, centered at $48.9^{\circ} \mathrm{C}$. The second one was a major sharp endothermic phase transition, from rippled $\left(\mathrm{P}_{\beta}\right)$ to liquid crystalline phase $\left(\mathrm{L}_{\alpha}\right)$, with peak at $53.6^{\circ} \mathrm{C}$. These thermotropic results are in line with past studies [19].

The pretransition peak, from gel $\left(\mathrm{L}_{\beta}\right)$ to ripple phase $\left(\mathrm{P}_{\beta}\right)$, vanished when polymer chains, regardless of their concentration, were entrapped into the lipid bilayers. As a consequence, it is suggested that the existence of $\mathrm{C}_{12} \mathrm{H}_{25}$-PNIPAM-COOH on the liposome outer surface 


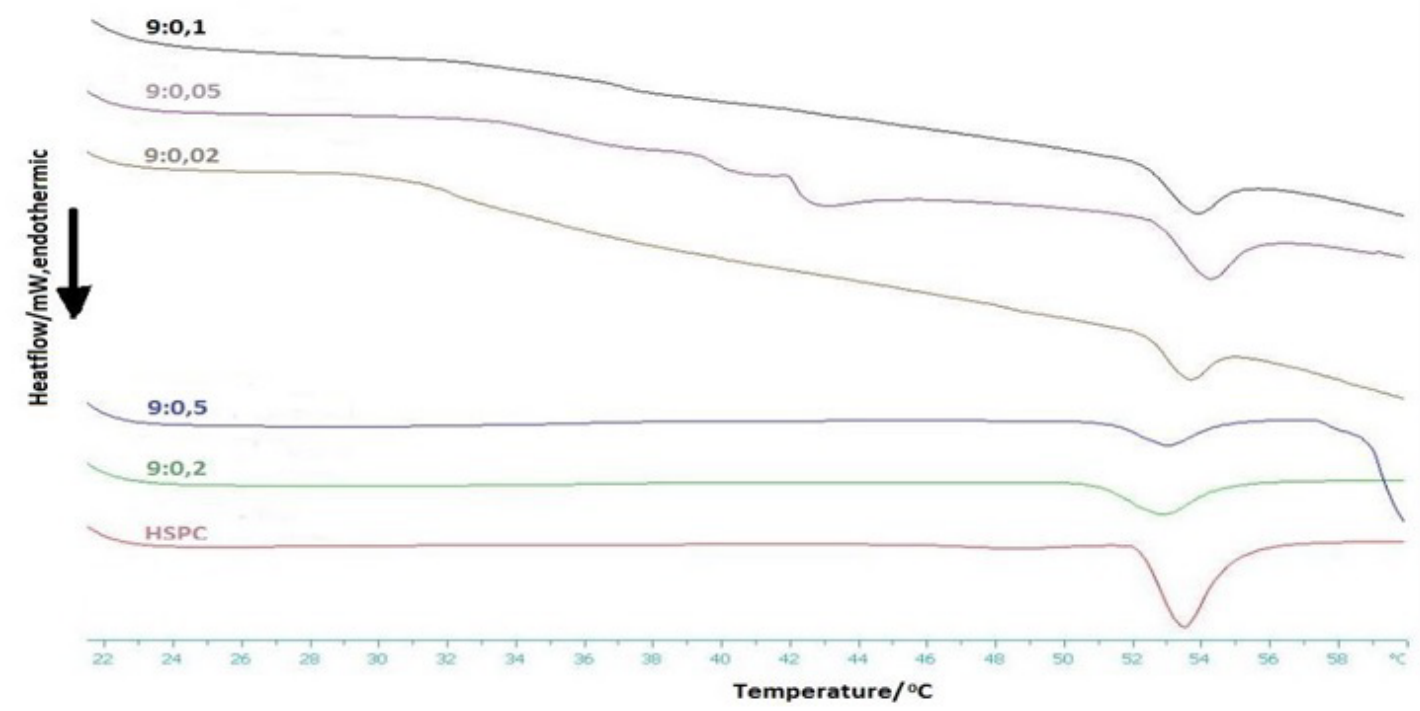

Figure 3. DSC heating scan of HSPC: $\mathrm{C}_{12} \mathrm{H}_{25}$-PNIPAM-COOH chimeric bilayers in PBS (pH=7.4) (Heating).

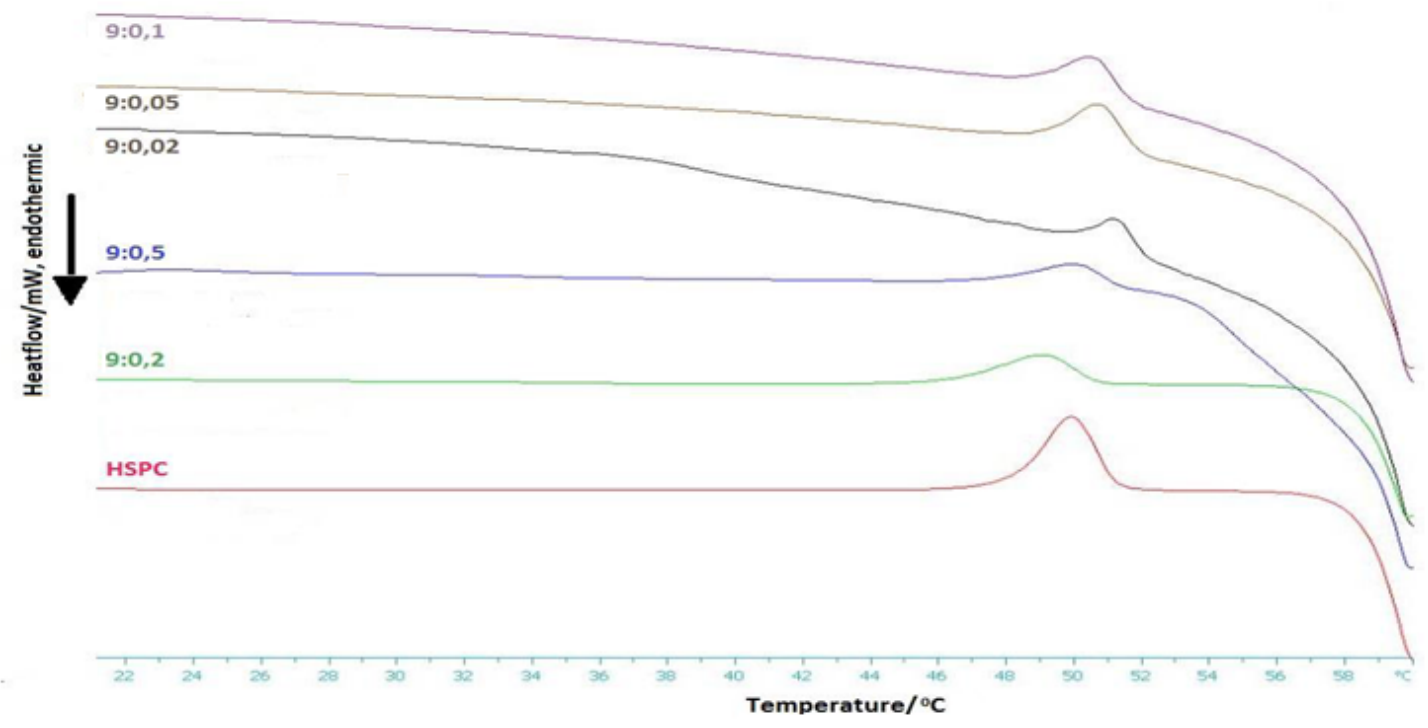

Figure 4. DSC cooling scan of HSPC: $\mathrm{C}_{12} \mathrm{H}_{25}$-PNIPAM-COOH chimeric bilayers in PBS ( $\mathrm{pH}=7.4$ ) (Cooling).

affects significantly the polar head group behavior.

As far as DSC results are concerned, we have likewise noticed two groups. Systems with lower molar ratios, 9:0.02, 9:0.05 and 9:0.1, kept absorbing energy above $32^{\circ} \mathrm{C}$, which is the polymer's lower critical solution temperature (LCST). The polymer chains turn hydrophobic, over $32^{\circ} \mathrm{C}$, due to their thermo-responsiveness, perturbate the lipid membrane and set the thermotropic parameters unable to be measured. This happens because of the hydrophobic tail $\mathrm{C}_{12} \mathrm{H}_{25}$, which penetrates into the bilayer and causes membrane disruption and phase separation. As a result, they exhibit properties similar to those of ordinary (isotropic) liquids $[14,20]$. However, a transition peak around $54^{\circ} \mathrm{C}$ was observed for each thermogram, which is the main transition temperature for HSPC lipids, something which is expected, as long as there is still conformation among phospholipids.

Molar ratios 9:0.2 and 9:0.5 belong to the second group. The thermoresponsive polymer altered the systems' thermotropic behavior and modified their main transition, compared to the one of pure HSPC lipid bilayers. More specifically, the specific enthalpy $\left(\Delta \mathrm{H}_{\mathrm{m}}\right)$ was substantially decreased, while the onset $\left(\mathrm{T}_{\text {onset, }}\right)$ and peak $\left(\mathrm{T}_{\mathrm{m}}\right)$ temperatures of the main transition remained almost unaffected, undergoing a downshift of less than $1^{\circ} \mathrm{C}$. Finally, the width at half peak height $\left(\Delta \mathrm{T}_{1 / 2}\right)$ slightly increased. These alterations prove that polymer chains cooperate well with HSPC lipids and that they do not induce significant fluidization [21].

After analyzing all of the thermograms, we conclude that lower molar ratios may not present an adequate thermotropic behavior, because they need longer relaxation time so as to achieve better cooperativity between polymer chains and phospholipids, a fact that is confirmed by their physicochemical studies.

Finally, the slight hysteresis in the cooling diagrams of both 
conventional and chimeric systems, around $3^{\circ} \mathrm{C}$, possibly suggests the formation of a partial interdigitated phase [20].

\section{Conclusion}

Conventional liposomes can be modified to produce advanced drug delivery nanosystems (aDDnSs), after the incorporation of stimulisensitive polymers, such as the thermoresponsive homopolymer $\mathrm{C}_{12} \mathrm{H}_{25}$ PNIPAM-COOH. The polymer guest decreases the membrane tension and prevents liposomal fusion at the same time. The present study aims to highlight that the different ratios of the polymer guest affect the properties and stability of these lyotropic liposomal dispersions in different ways. Regarding HSPC: $\mathrm{C}_{12} \mathrm{H}_{25}$-PNIPAM-COOHmixed liposomes, physicochemical studies prove that the most stable systems are 9:0.1 and 9:0.2 molar ratios. These systems not only maintain their hydrodynamic diameter in the long run of one month, but they were also found to be homogeneous enough, due to steric/enthalpic stabilization. Thermodynamic findings have also indicated that better cooperativity was developed with higher concentrations of $\mathrm{C}_{12} \mathrm{H}_{25}-\mathrm{PNIPAM}-\mathrm{COOH}$, where the polymer slightly affected the thermotropic behavior of the lipid bilayer. These results may be taken as a trigger for developing innovative liposomal formulations, incorporating drugs improving their pharmacokinetic profile and consequently their effectiveness, taking into consideration the nanocarriers' stimuli responsiveness.

\section{References}

1. Lagerwall JPF, Scalia G, Nguyen TQ, Wu JJ, Doan V, et al. (2012) A new era for liquid crystal research: Applications of liquid crystals in soft matter nano-, bio- and microtechnology. Curr Appl Phys 12: 252-268.

2. Shashi K, Satinder K, Bharat P (2012) A complete review on: Liposomes. Int J Pharm 7:10-16.

3. Gin DL, Pecinovsky CS, Bara JE, Kerr RL (2008) Functional Lyotropic Liquid Crystal Materials. Struct Bond 128: 181-222.

4. Hoffman AS (1995) "Intelligent" polymers in medicine and biotechnology. Artif Organs 19: 458-467. [Crossref]

5. Ward MA, Georgiou TK (2011) Thermoresponsive Polymers for Biomedical Applications. Polymers 3: 1215-1242.

6. Roy D, Brooks WL, Sumerlin BS (2013) New directions in thermoresponsive polymers. Chem Soc Rev 42: 7214-7243. [Crossref]
7. Demetzos C, Pippa N (2014) Advanced drug delivery nanosystems (aDDnSs): a minireview. Drug Deliv 21: 250-257. [Crossref]

8. Kono K, Nakai R, Morimoto K, Takagishi T (1999) Thermosensitive polymer-modified liposomes that release contents around physiological temperature. Biochim Biophys Acta 1416: 239-250. [Crossref]

9. Pippa N, Meristoudi A, Pispas S, Demetzos C (2015) Temperature-dependent drug release from DPPC:C12H25-PNIPAM-COOH liposomes: Control of the drug loading/ release by modulation of the nanocarriers' components. Int J Pharm 485: 374-382. [Crossref]

10. Joglekar M, Trewyn BG (2013) Polymer-based stimuli-responsive nanosystems for biomedical applications. Biotechnol J 8: 931-945. [Crossref]

11. Mura S, Nicolas J, Couvreur P (2013) Stimuli-responsive nanocarriers for drug delivery. Nat Mater 12: 991-1003. [Crossref]

12. Hadinoto K, Sundaresan A, Cheow WS (2013) Lipid-polymer hybrid nanoparticles as new generation therapeutic delivery platform: a review. Eur J Pharm Biopharm 85: 427-443. [Crossref]

13. Ganta S, Devalapally H, Shahiwala A, Amiji M (2008) A review of stimuli-responsive nanocarriers for drug and gene delivery. J Control Release 126: 187-204. [Crossref]

14. Naziris N, Pippa N, Meristoudi A, Pispas S, et al. (2017) Design and development of pH-responsive HSPC:C12H25-PAA chimeric liposomes. J Liposome Res 27: 108-117. [Crossref]

15. Tribet C, Vial F (2008) Flexible macromolecules attached to lipid bilayers: impact on fluidity, curvature, permeability and stability of the membranes. Soft Matter 4: 68-81.

16. Lutz JF, Akdemir O, Hoth A (2006) Point by point comparison of two thermosensitive polymers exhibiting a similar LCST: is the age of poly(NIPAM) over? J Am Chem Soc 128: 13046-13047. [Crossref]

17. Immordino ML, Dossio F, Cattel L (2006) Stealth liposomes: review of the basic science, rationale, and clinical applications, existing and potential. Int J Nanomedicine 1: 297-315. [Crossref]

18. Derjaguin B, Landau L (1993) Theory of the stability of strongly charged lyophobic sols and of the adhesion of strongly charged particles in solutions of electrolytes. Prog Surf Sci 1: 30-59.

19. Koynova R, Caffrey M (1998) Phases and phase transitions of the phosphatidylcholines. Biochim Biophys Acta 1376: 91-145. [Crossref]

20. Demetzos C (2008) Differential Scanning Calorimetry (DSC): A Tool to Study the Thermal Behavior of Lipid Bilayers and Liposomal Stability. J Liposome Res 18: 159173. [Crossref]

21. Kolman I, Pippa N, Meristoudi A, Pispas S, Demetzos C (2016) A dual-stimuliresponsive polymer into phospholipid membranes-A thermotropic approach. $J$ Therm Anal Calorim 123: 2257-2271.

Copyright: (C2017 Tzani A. This is an open-access article distributed under the terms of the Creative Commons Attribution License, which permits unrestricted use, distribution, and reproduction in any medium, provided the original author and source are credited. 